\title{
Significance of new blood vessels in the pathogenesis of temporomandibular joint osteoarthritis
}

\author{
JIANLIN LIU, JUAN DAI, YANSONG WANG, SIYU LAI and SUWEN WANG \\ Department of Stomatology, Shenzhen Hospital of Guangzhou University \\ of Chinese Medicine, Shenzhen, Guangdong 518033, P.R. China
}

Received June 21, 2016; Accepted January 19, 2017

DOI: 10.3892/etm.2017.4234

\begin{abstract}
We studied the significance of new blood vessels in the pathogenesis of temporomandibular joint osteoarthritis (TMJOA). Fifteen 8-week-old female Sprague-Dawley rats were selected to establish TMJOA models of gradually induced occlusal disorders. Five rats were sacrificed at 4,8 and 16 weeks, and histological exam was conducted along with micro-computed tomography observation on the condyle specimen. The distribution and number of new blood vessels breaking were observed through the tidemark through CD34 immunofluorescence staining. The proliferation of chondrocytes were detected through Ki67 immunohistochemical staining, and the differentiation functions of chondrocytes were observed through PTHrP and IHH immunohistochemical staining. The degradation functions of cartilage matrix were observed through matrix metalloproteinase (MMP)-9 immunohistochemical staining to detect the expression of vascular growth promotion and inhibition factors with vascular endothelial growth factor (VEGF), CTGF and CHM-1 immunohistochemical staining and screen differentially expressed genes through gene chip analysis method. It was found that the condyle tissue full thickness, fiber layer thickness and calcified cartilage layer thickness were significantly increased with time $(\mathrm{P}<0.05)$. Bone mineral density, trabecular thickness and Tb.Sp were also increased significantly with time, BS/BV and trabecular number were decreased significantly with time $(\mathrm{P}<0.05)$. The new blood vessels reached the deep layer of calcified cartilage until the tide line was broken and non-calcified cartilage was invaded. The number of vessels were increased significantly with time $(\mathrm{P}<0.05)$. Ki67, PTHrP and IHH-positive rates were
\end{abstract}

Correspondence to: Dr Jianlin Liu, Department of Stomatology, Shenzhen Hospital of Guangzhou University of Chinese Medicine, 6001 Beihuan Road, Futian, Shenzhen, Guangdong 518033, P.R. China

E-mail: jianlin_liu12@163.com

Key words: new blood vessels, temporomandibular joint osteoarthritis, CD34, Ki67, PTHrP, IHH, matrix metalloproteinase, vascular endothelial growth factor, CTGF, CHM-1, gene chip increased significantly $(\mathrm{P}<0.05)$. MMP-9, VEGF, CTGF and CHM-1 were increased significantly $(\mathrm{P}<0.05)$. VEGF, CTGF and CHM-1 mRNA were upregulated differentially with the expressed genes. In conclusion, the new blood vessels may be important in the pathogenesis of TMJOA.

\section{Introduction}

Temporomandibular joint osteoarthritis (TMJOA) is the most serious category in temporomandibular joint disorder disease in the oral and maxillofacial region, which can lead to severe joint pain and joint movement disorder and seriously damage the life quality of the patient. The pathological characteristics of TMJOA mainly include condylar cartilage degeneration and abnormal subchondral bone sclerosis (1). The subchondral bone remodeling cannot only change the stress characteristics of subchondral bone, but the new blood vessels at the boundary of the cartilage and subchondral bone can also carry factors to influence chondrocyte metabolism through 'dialogue' (2). The preliminary studies found that under normal physiological conditions, the expression of hypertrophic chondrocytes of rat condylar cartilage can promote angiogenic factor vascular endothelial growth factor (VEGF) and inhibit anti-angiogenic factor CHM-1, and its expression quantity decreased with age (3). The overexpression of VEGF can promote proliferation of chondrocytes and the deposition of new bones under the cartilage, and if the matrix synthesis is greater than the degradation, the growth of the mandible will be promoted. However, under the action of abnormal bite force, the condylar cartilage of TMJOA rats will be degraded, the ubchondral bone will be lost and a large amount of the inflammatory factor and matrix metalloproteinase (MMP) will be secreted. At the osteochondral boundary, VEGF and CTGF can promote high expressions of angiogenic factor and the number of new blood vessels will be increased significantly, breaking through the tidemark and reaching calcified and non-calcified cartilage.

There are still some issues to be investigated e.g., type of pro-angiogenic factors to induce the formation of new blood vessels under the action of the abnormal force and whether there is a synergistic effect between it and VEGF, role of pro-angiogenic factors in the chondrocyte differentiation and apoptosis under the action of the abnormal force, role of antergic pro-angiogenic factors in inhibiting the cartilage 
degradation and osteophyte formation. The answer to these issues can further clarify the pathogenesis of TMJOA lesions and be more conducive when looking for effective drug targets for the treatment of TMJOA. To this end, the study will explore the significance of new blood vessels in the pathogenesis of TMJOA by constructing animal models.

\section{Materials and methods}

Animals model. Fifteen 8-week-old female Sprague-Dawley (SD) rats with the average weight of $\sim 200 \mathrm{~g}$ were kept under conventional breeding; $3 \mathrm{M}$ orthodontic apron (no. 1/8; G\&H Orthodontics, Franklin, IN, USA). The TMJOA models were established in gradually induced occlusal disorder. The animals were anesthetized and the orthodontic apron was placed between the first and the second molars in the lower right and upper left positions. The mesial movement of the first molar were made by nearly $1 \mathrm{~mm}$ after 1 week, and then the apron was removed and placed in self-curing plastics in the gap so as to make it below the occlusal plane. Subsequently, the same operations was repeated between the second and third molars at the lower right and upper left position at 4 weeks after the first separation so as to make the distal movement of the third molar. Next, 5 rats were sacrificed at 4,8 and 16 weeks after successful modeling, and TMJ was removed at the right side to fix it with $4 \%$ paraformaldehyde for $48 \mathrm{~h}$. It was followed by decalcification with sodium formate for 1 week, then the decalcification solution was cleaned-up, graded dehydration with ethanol was performed, then embedded with paraffin, the sections were cut longitudinally in proximal, distal and immediate directions to make condylar specimens of $5 \mu \mathrm{m}$. The approval for animal experiments was received from the Animal Ethics Committee of Guangzhou University Animal Center.

Study method. Histological and micro-computed tomography (Micro-CT) observations were performed on the condyle specimens. The distribution and number of new blood vessels breaking were observed through the tidemark through CD34 immunofluorescence staining. The proliferation condition of chondrocytes were observed through Ki67 immunohistochemical staining, the differentiation functions of chondrocytes were conducted through PTHrP and IHH immunohistochemical staining. The degradation functions of cartilage matrix were measured through MMP-9 immunohistochemical staining. The expression of vascular growth promotion and inhibition factors with VEGF, CTGF and CHM-1 immunohistochemical staining and then screened differentially expressed genes through gene chip analysis method (Fig. 1).

Histological observation. For conventional hematoxylin and eosin (H\&E) staining, Leica DFC490 system (Leica Microsystems, Wetzlar, Germany) was used for collection of microscopic images. The condyle surface was divided into the front, middle and rear parts with articular disc front and rear joint as the boundary, quarter each part. The Leica QWin software (Leica Microsystems Imaging Solution, Ltd., Cambridge, UK) was applied to measure the fiber layer thickness, calcified cartilage layer thickness and full-layer cartilage thickness at a total of 9 positions in each quartering point and then taking the mean value for analysis. The Micro-CT (GE Healthcare, London, ON, Canada) was used to scan the specimen with the resolution of $8 \mu \mathrm{m}$ and the peak voltage of $80 \mathrm{kV}$. Microview software (version 2.5.0; GE Healthcare) was used to re-construct the condylar specimen axial, coronal and sagittal images and to calculate the bone mineral density (BMD) and bone microstructure parameters [bone surface area-to-volume ratio (BS/BV), trabecular number (Tb.N), trabecular thickness (Tb.Th) and trabecular bone gap (Tb.Sp)] of each specimen. The $0.5 \times 0.5 \times 0.5 \mathrm{~mm}$ region of interest (ROI) at the midpoint of the condyle postmedian was selected and just below the boundary of the cartilage and subchondral bone software was used to automatically calculate subchondral bone-related parameters in the region of interest.

CD34 immunofluorescence staining. CD34 polyclonal antibody (Santa Cruz Biotechnology, Inc., Santa Cruz, CA, USA), immunofluorescence kit (Zhongshan Biologics Co., Zhongshan, China) was used. The tissue slides were placed for antigen digestion at $37^{\circ} \mathrm{C}$ for $10 \mathrm{~min}$ after xylene dewaxing and graded alcohol dehydration. The slides were washed with phosphate-buffered saline (PBS) for $5 \mathrm{~min}, 3$ times and placed in the antigen retrieval solution at $37^{\circ} \mathrm{C}$ for $15 \mathrm{~min}$. Subsequently, the sides were washed with PBS for 5 min, 3 times, and incubated in serum at $37^{\circ} \mathrm{C}$ for $20 \mathrm{~min}$. After discarding serum, the sections were covered with CD34 primary antibody (1:50) at $4{ }^{\circ} \mathrm{C}$ overnight. It was followed by washing the slide with PBS for $5 \mathrm{~min}, 3$ times, and addition of fluorescently labeled secondary antibody $\mathrm{Cy} 3$ to incubate at $37^{\circ} \mathrm{C}$ for $60 \mathrm{~min}$. It was again followed by washing with PBS for $5 \mathrm{~min}, 3$ times, and slides were fixed with glycerol to take an image by confocal microscopy.

Ki67 immunohistochemical staining. Ki67 monoclonal antibody (1:200 dilution, Lab Vision Corp., Fremont, CA, USA) was used. The staining was performed as previously described (4). Likewise, immunohistochemical staining was performed for PTHrP, IHH, MMP-9, VEGF, CTGF and CHM-1 with monoclonal antibodies (1:200 dilution; Sigma-Aldrich, St. Louis, MO, USA).

Gene chip analysis method. HumanHT-12 v4 Expression BeadChip (Illumina, Inc., San Diego, CA, USA) was used. Total RNA was extracted and purified, micro-sample in vitro amplification, probe labeling and hybridization and chip scanning were performed to obtain the original signal value of each gene point on the chip. The Database for Annotation, Visualization and lntegrated Discovery (DAVID) analysis tool was used to screen the difference in expression genes. The genes with more than 1.5 times expression change (i.e., the genes with the fold value $>1.5$ or $<0.67$ ) was considered as differentially expressed genes according to the signal intensity ratio. The screened different mRNA were presented with logarithmic scatter diagram and was divided along the red diagonal line. The upper was a point with the fold $>1.5$, the lower was the point with fold $<0.67$ and the blue represented differentially expressed genes.

Statistical analysis. SPSS 19.0 statistical software (SPSS, Inc., Chicago, IL, USA) was used for entry and data analysis. The quantitative data were expressed as mean \pm standard deviation and single factor analysis of variance (ANOVA) was used for the data analysis in terms of the comparison between groups. 


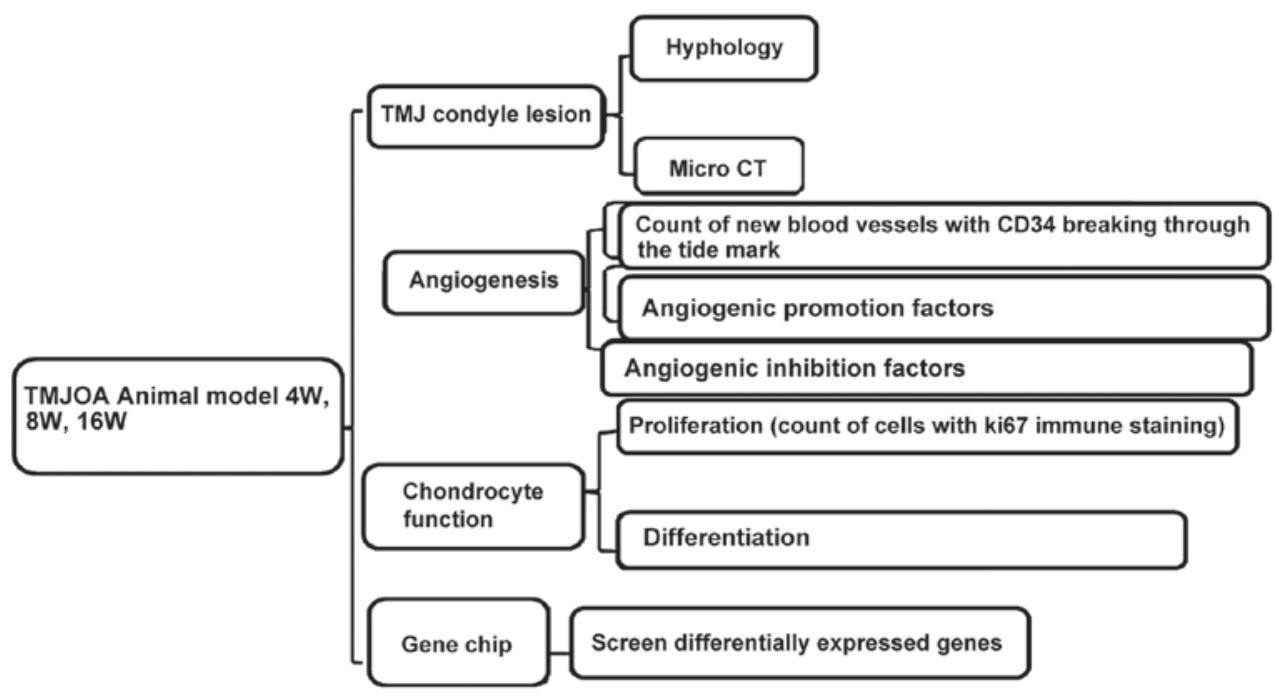

Figure 1. Test flow chart.

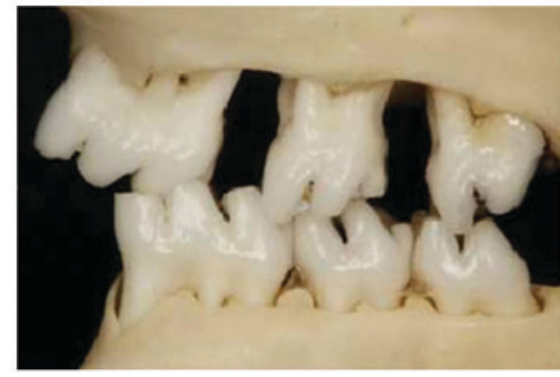

Figure 2. TMJOA model. TMJOA, temporomandibular joint osteoarthritis.

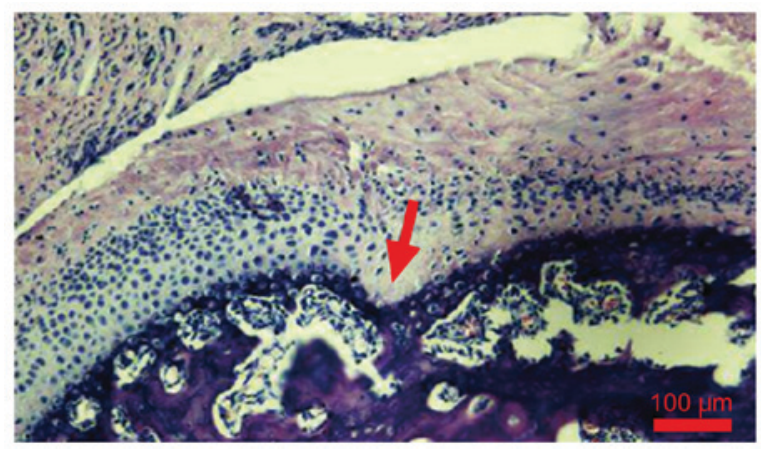

Figure 3. Condyle tissues full thickness, fiber layer thickness and calcified cartilage layer thickness increased with time.

$\mathrm{P}<0.05$ was considered to indicate a statistically significant difference.

\section{Results}

Model of histological observation and Micro-CT quantitative detection. After respective mesial and distal movement of the first and third molars of rats in the lower right and upper left positions, the original relationship between the sharpness and concaveness was destroyed and an experimental occlusal disorder was formed (Fig. 2). The fibrous layer structure of the condyle surface was incomplete, the chondrocytes were

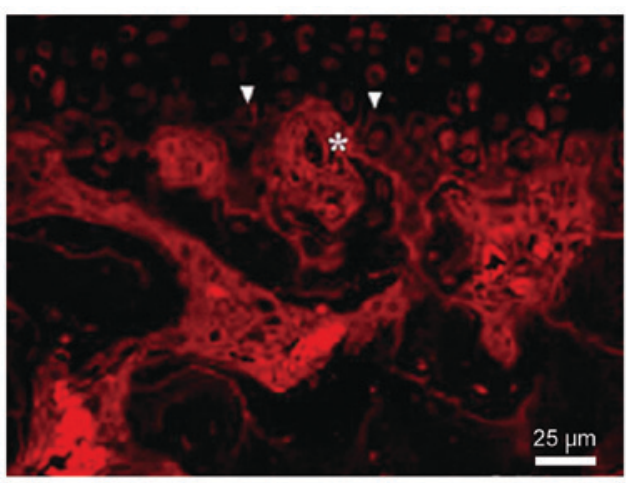

Figure 4. Condyle CD34 immunofluorescence staining (the arrow shows the tide line and the asterisk shows the vessel labeled with CD34).

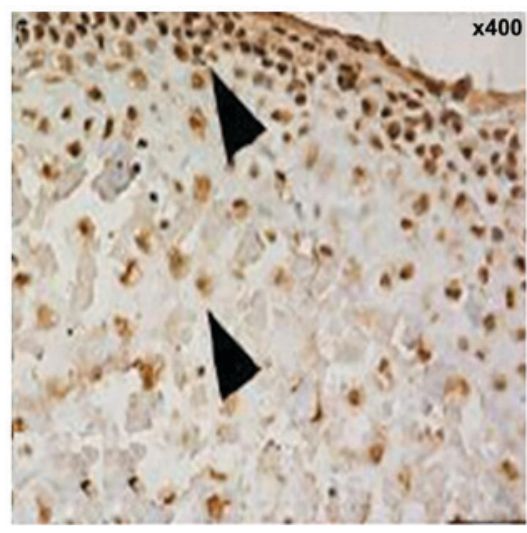

Figure 5. Condylar cartilage Ki67.

in disorder, and it could be seen that the mast cell clusters were integrated in an island shape, the chondrocyte nail-like hyperplasia was protruded into the subchondral bone, there was an acellular region, tangent crack was formed and similar osteophyte-like hyperplasia occurred. The lesions were mainly concentrated in the middle and rear parts of the condylar cartilage, and under the observation by microscope, the tide line showed a basophilic wavy line, which clearly separated the 
Table I. Histological condyle tissue observation $(\mu \mathrm{m})$.

\begin{tabular}{lccrr}
\hline Items & 4 weeks & 8 weeks & 16 weeks & F-value \\
\hline Full-layer thickness & $166.4 \pm 23.5$ & $183.2 \pm 26.8$ & $221.7 \pm 30.3$ & 6.569 \\
Fiber layer thickness & $45.5 \pm 7.2$ & $51.2 \pm 7.6$ & $56.8 \pm 7.7$ & 6.238 \\
Calcified cartilage & $71.4 \pm 8.3$ & $78.2 \pm 8.5$ & $84.4 \pm 8.6$ & 6.425 \\
layer thickness & & & & 0.012 \\
\hline
\end{tabular}

Table II. Micro-CT scanning of condyle tissue.

\begin{tabular}{|c|c|c|c|c|c|}
\hline Items & 4 weeks & 8 weeks & 16 weeks & F-value & P-value \\
\hline $\operatorname{BMD}\left(\mathrm{mg} / \mathrm{cm}^{3}\right)$ & $412.3 \pm 56.5$ & $526.4 \pm 62.3$ & $729.2 \pm 75.8$ & 12.532 & $<0.001$ \\
\hline $\mathrm{BS} / \mathrm{BV}(\mathrm{mm})$ & $42.5 \pm 4.7$ & $33.6 \pm 4.2$ & $25.2 \pm 3.3$ & 10.527 & $<0.001$ \\
\hline Tb.N (mm) & $11.5 \pm 2.3$ & $8.6 \pm 1.7$ & $6.2 \pm 1.4$ & 8.624 & $<0.001$ \\
\hline Tb.Th (mm) & $0.04 \pm 0.01$ & $0.06 \pm 0.02$ & $0.10 \pm 0.03$ & 9.466 & $<0.001$ \\
\hline Tb.Sp (mm) & $0.05 \pm 0.01$ & $0.06 \pm 0.01$ & $0.07 \pm 0.01$ & 7.452 & $<0.001$ \\
\hline
\end{tabular}

Micro-CT, micro-computed tomography; BS/BV, bone surface/bone volume; Tb.N, trabecular number; Tb.Th, trabecular thickness.

Table III. Comparison of the number of new blood vessels (vessel/HP).

\begin{tabular}{|c|c|c|c|c|c|}
\hline Items & 4 weeks & 8 weeks & 16 weeks & F-value & P-value \\
\hline $\begin{array}{l}\text { Number of blood vessels ending } \\
\text { in the calcified cartilage layer }\end{array}$ & $15.3 \pm 4.2$ & $17.2 \pm 4.6$ & $19.6 \pm 4.8$ & 7.629 & $<0.001$ \\
\hline $\begin{array}{l}\text { Number of blood vessels ending } \\
\text { in the non-calcified cartilage layer }\end{array}$ & $1.5 \pm 0.3$ & $2.2 \pm 0.5$ & $3.7 \pm 0.9$ & 9.432 & $<0.001$ \\
\hline
\end{tabular}

Table IV. Positive expression rates of MMP-9, VEGF, CTGF and CHM-1 (\%).

\begin{tabular}{lccccc}
\hline Items & 4 weeks & 8 weeks & 16 weeks & F-value & P-value \\
\hline MMP-9 & $5.3 \pm 1.1$ & $6.7 \pm 1.2$ & $8.2 \pm 1.3$ & 7.451 & $<0.001$ \\
VEGF & $3.2 \pm 0.5$ & $3.6 \pm 0.7$ & $4.4 \pm 0.8$ & 6.957 & $<0.001$ \\
CTGF & $4.3 \pm 0.6$ & $4.5 \pm 0.7$ & $4.9 \pm 0.8$ & 5.865 & $<0.001$ \\
CHM-1 & $4.5 \pm 1.2$ & $6.6 \pm 1.4$ & $8.3 \pm 1.6$ & 7.702 & $<0.001$ \\
\hline
\end{tabular}

MMP, matrix metalloproteinase; VEGF, vascular endothelial growth factor.

calcified cartilage from the non-calcified cartilage. The condyle tissue full thickness, fiber layer thickness and calcified cartilage layer thickness were increased with the time and the difference was statistically significant $(\mathrm{P}<0.05)$ (Fig. 3 and Table I). BMD, Tb.Th and Tb.Sp increased with time, BS/BV and Tb.N were decreased with time and the difference was statistically significant $(\mathrm{P}<0.05)$ (Table II).

Distribution and number of new blood vessels. The vessel could reach the deep layer of the calcified cartilage until the breakthrough of the tide line and the invasion of non-calcified cartilage. The number of vessels was increased significantly with time $(\mathrm{P}<0.05)$ (Fig. 4 and Table III).

The proliferationand differentiationfunctions of chondrocytes. Ki67 showed brown, or brown nuclei, which were gradually extended from the fiber layer and the proliferation layer to the maturity layer and hypertrophic layer. The proliferation rate was increased with time and the difference was statistically significant $(12.5 \pm 3.3,15.7 \pm 3.6$ and $18.4 \pm 3.7 \%, F=7.648$, $\mathrm{P}<0.001$ ) (Fig. 5). PTHrP and IHH were distributed mainly in shallow layer of condylar cartilage hypertrophy, the positive rate was increased with time and the difference was statistically significant $(22.4 \pm 3.6,35.6 \pm 5.2$ and $56.4 \pm 7.3 \%, F=11.427$, $\mathrm{P}<0.001) ;(16.7 \pm 3.2,22.5 \pm 4.4$ and $43.6 \pm 6.6 \%), \mathrm{F}=10.326$, $\mathrm{P}<0.001$ ) (Fig. 6).

Degradation functions of cartilage matrix and expression of vascular growth promotion and inhibition factor. MMP-9, VEGF and CTGF were mainly expressed in the cartilage proliferative layer and hypertrophy layer, the brown staining was mainly seen in the nucleus and cytoplasm. The strong CHM-1 expression was in condylar cartilage full layer and was clearly expressed in the hypertrophy cartilage deep 

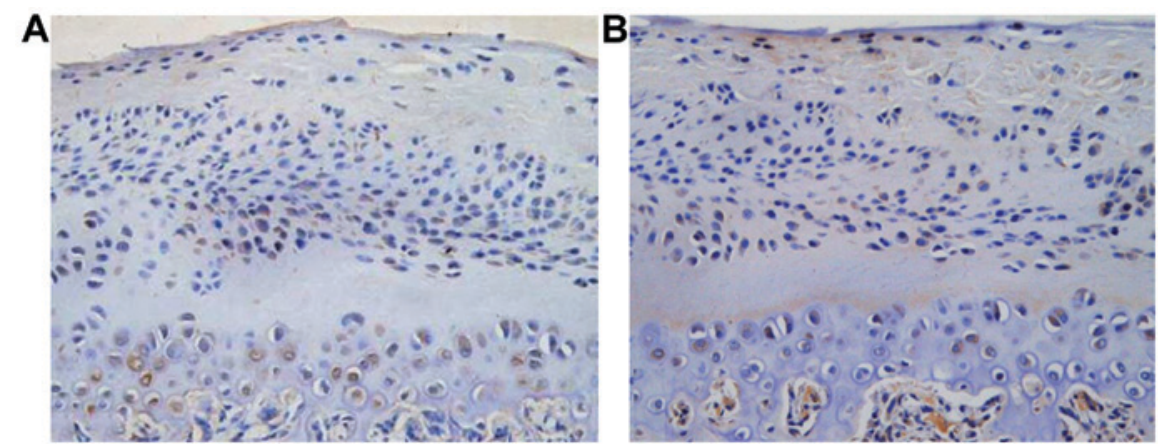

Figure 6. PTHrP and IHH immunohistochemical staining (x200) (A, PTHrP and B, IHH). Immunohistochemical staining (the arrow shows expression of Ki67 in the proliferation layer, fibrous layer and hypertrophic layer).
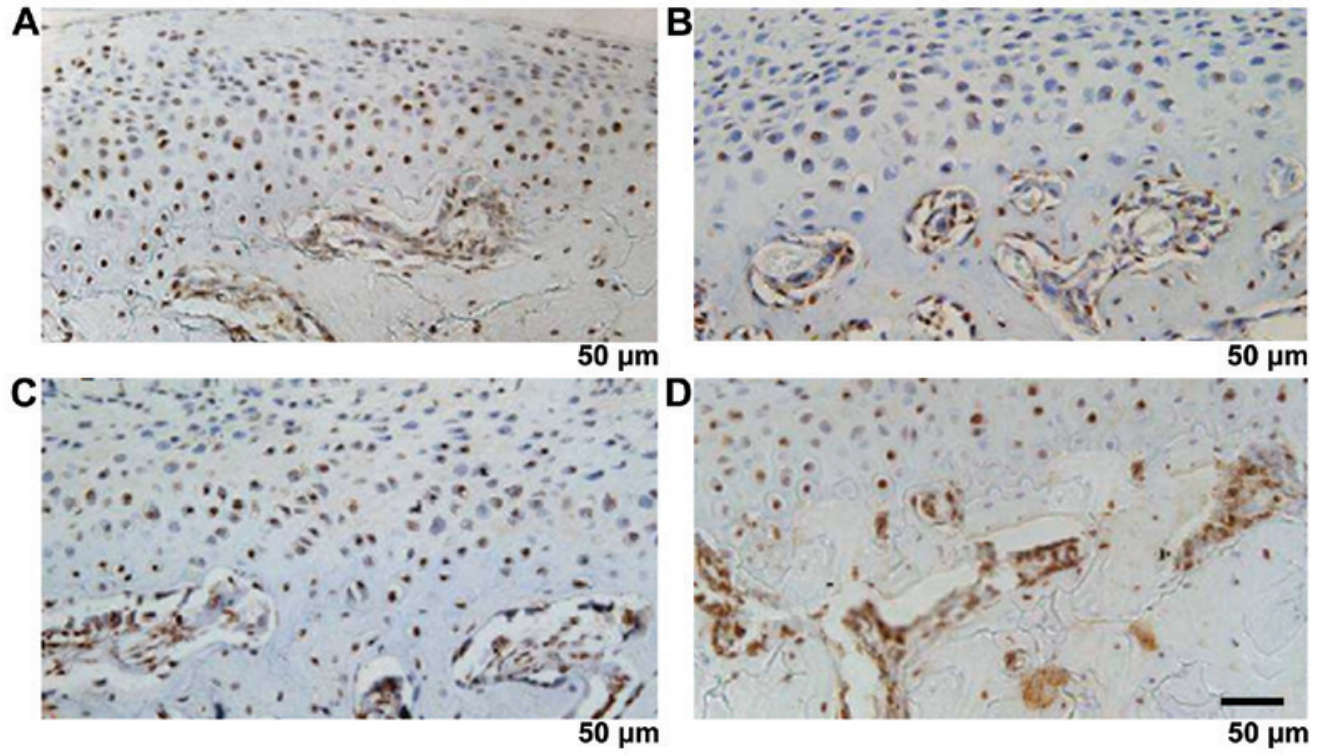

Figure 7. (A) MMP-9, (B) VEGF, (C) CTGF and (D) CHM-1 immunohistochemical staining. MMP, matrix metalloproteinase; VEGF, vascular endothelial growth factor.

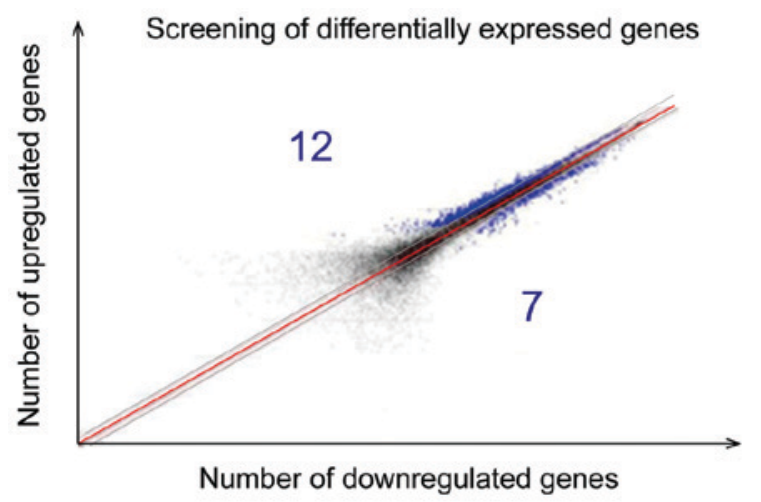

Figure 8 . The screening of differentially expressed genes by gene chip analysis method.

layer close to the bone cartilage boundary. MMP-9, VEGF, CTGF and CHM-1 were increased significantly with time $(\mathrm{P}<0.05)$ (Fig. 7 and Table IV).

Screening of differentially expressed genes by gene chip analysis method. TMJOA can upregulate 12 kinds of differ- entially expressed genes and downregulate seven kinds of differentially expressed genes, among which VEGF, CTGF and CHM-1 mRNA can upregulate the differentially expressed genes (Fig. 8).

\section{Discussion}

The new blood vessel of condylar cartilage plays an important role in the occurrence and development of TMJOA, elucidating that its mechanism has important theoretical significance and clinical guidance effect. There are no blood vessels in the normal condylar cartilage, and its physiological metabolism relies on the support of the synovial fluid and adjacent tissues containing rich blood vessels, while the stability of such a system is essential for the joint tissue integrity and strong biological force with standing. Under normal physiological conditions, the articular cartilage has the ability to resist the invasion of blood vessels, but OA cartilage seems to have lost such ability (5). The early blood vessels of OA from subchondral bone can be invaded into the calcified cartilage and can gradually spread to the cartilage of the superficial layer with the progression of the disease. The blood vessel 
invasion can destroy the integrity of the boundary of articular cartilage and subchondral bone, the vascular endothelial cells can secrete a variety of factors simultaneously, thereby regulating the growth and metabolism of cartilage cells and may directly involve in the degradation of cartilage matrix (6). It has been believed that the thinning of OA articular cartilages is not only associated with the degeneration of the cartilage tissue itself, but also associated with the bone formation in the cartilage proceeded continuously at the boundary of bone and cartilage, while the angiogenesis is an important condition for endochondral bone formation and osteophyte formation (7). Recent studies also showed that the level of vascularization of the articular cartilage may reflect the degeneration of cartilage and severity of OA clinical symptoms (8). Joint pain is considered to be an important feature of OA, and there are no neural tissues in the cartilage under normal conditions. But with the destruction of bone-cartilage boundary, the sensory nerves may be invaded into the cartilage tissue with new blood vessels and simultaneously such nerve tissue that are growing more sensitive to pain (9). If the angiogenesis of the articular cartilage is inhibited, the pain symptom of OA will also be greatly reduced. Therefore, the study on the new blood vessels between the articular cartilage and subchondral bone under TMJOA and the finding of the key link and key signal transduction molecules therein is greatly significant for in-depth understanding of the occurrence and development TMJOA.

TMJOA condylar cartilage vascular invasion is a complex process involving multiple-cell proliferation, migration, degradation, extracellular matrix degradation and remodeling and may be associated with the disequilibrium of factors regulating blood vessels, which illustrates the angiogenic factor promotion and inhibition role and is regarded as an important breakthrough for studying the TMJOA articular cartilage angiogenesis. The reason for the start of vascular invasion leading to cartilage degeneration are still unclear. It is speculated that due to the effect of the abnormal stress, the blood vessel can break through the calcified cartilage and tidemark and damage the microenvironment of articular cartilage independent from the subchondral bone (10). On the other hand, the intravascular cells can secrete various factors and cause damage on the chondrocyte normal physiological functions (11). Further, the vascular invasion is also a physical disruption for the normal arrangement of the chondrocyte and collagen fibers (12). The in vitro experiment also discloses that the vascular endothelial cells can generate a hypertrophy promotion effect by cascade reaction similar to the complement system through the secretion of serine proteases, cysteine proteases and aspartic proteases (13). It can be seen that the vascular invasion may promote the cartilage calcification or ossification and the vascular endothelial cells may regulate the functions of cartilage cells and promote the degradation of the cartilage matrix. The condylar cartilage can inhibit the disability of the vascular invasion mechanism, including promoting the angiogenic promotion factor expression upregulation and angiogenic inhibition factor expression downregulation or loss of expression may be one of the key links. The studies have shown that there is a high expression of VEGF in OA articular cartilage (14). VEGF is an angiogenic promotion factor with powerful roles, which may directly or indirectly be involved in every link of angiogenesis. VEGF is also considered as an important regulatory factor during the condylar cartilage growth and development. Under normal physiological conditions, the hypertrophic chondrocyte can secrete VEGF, promote the invasion of new blood vessels and raise a lot of broken cartilage chondrocytes to absorb the cartilage, while it may be also accompanied by a large number of osteoblasts, promoting new bone deposition. However, the OA cartilage cells under lesions can secrete VEGF, promote the adjacent normal cartilage cells to secrete MMP, interleukin-1 $\beta$ and other inflammatory cytokines (15). The stress change is one of the causes of OA, in rat models with TMJOA established in abnormal occlusal disorder, VEGF is mainly expressed in the chondrocytes of the mature layer and hypertrophic layer of the condylar cartilage and its expression is enhanced with the extension of the force application time. The increase in calcified layer osteoclasts may be associated with VEGF. In addition, the level of expression of articular cartilage VEGF in patients with OA is positively correlated with the cartilage vascularization density (16). It can be seen that VEGF may play a variety of biological effects such as promoting angiogenesis during the TMJOA development.

However, there is still a certain distance from the site where VEGF is expressed to the bone, the cartilage boundary, angiogenesis has synergistic effects on multiple factors and there may be other more important factors promoting angiogenesis. We also found that the blood vessel invasion is closely associated with the area losing collagen, which prompts that under abnormal stress, the change of cartilage matrix is conductive to the invasion of blood vessels into the articular cartilage. Therefore, the finding that there are more effective angiogenic promotion factors and collagen matrix degradation promotion factors in TMJ joints is helpful in clarifying the formation mechanism of TMJOA new blood vessels.

We showed that the condyle tissue full layer thickness, fiber layer thickness and calcified cartilage thickness were increased with time and the difference was statistically significant. BMD, Tb.Th and Tb.Sp were significantly increased with time, BS/BV and Tb.N were significantly decreased with time. The new blood vessels can reach the calcified cartilage deep layer until the breakthrough of the tide line and invasion into the non-calcified cartilage. The number of the blood vessels was increased with time and the difference was statistically significant. Ki67, PTHrP and IHH-positive rates were increased with time and the difference was statistically significant. MMP-9, VEGF, CTGF and CHM-1 were increased with time and the difference was statistically significant. VEGF, CTGF and CHM-1 mRNA were upregulated differentially expressed genes. This study did not set a control group, which has been described in previous studies in detail. The study mainly analyzed the formation and development process of the new blood vessels in TMJOA models over time, found the distribution characteristics and growth changes, proliferation and differentiation of chondrocytes and matrix degradation and changes in vascular growth factor promotion and inhibition and verified them through the differential gene expression. In comparison with other models of acute injury, TMJOA model was more consistent with the analysis on whether the better therapeutic effect was provided against TMJOA through target point intervention during the course of the body stress chronic disease progression. 
In conclusion, the new blood vessels may be important in pathogenesis of TMJOA and could be potential targets for intervention.

\section{References}

1. Bechtold TE, Saunders C, Decker RS, Um HB, Cottingham N Salhab I, Kurio N, Billings PC, Pacifici M, Nah HD, et al: Osteophyte formation and matrix mineralization in a TMJ osteoarthritis mouse model are associated with ectopic hedgehog signaling. Matrix Biol 52-54: 339-354, 2016.

2. Tanaka E, Aoyama J, Miyauchi M, Takata T, Hanaoka K, Iwabe $\mathrm{T}$ and Tanne K: Vascular endothelial growth factor plays an important autocrine/paracrine role in the progression of osteoarthritis. Histochem Cell Biol 123: 275-281, 2005.

3. Lingaraj K, Poh CK and Wang W: Vascular endothelial growth factor (VEGF) is expressed during articular cartilage growth and re-expressed in osteoarthritis. Ann Acad Med Singapore 39: 399-403, 2010

4. Hayashi Y, Takei H and Kurosumi M: Ki67 immunohistochemical staining: the present situation of diagnostic criteria. Nihon Rinsho 70 (Suppl 7): 428-432, 2012 (In Japanese).

5. Ludin A, Sela JJ, Schroeder A, Samuni Y, Nitzan DW and Amir G: Injection of vascular endothelial growth factor into knee joints induces osteoarthritis in mice. Osteoarthritis Cartilage 21: 491-497, 2013

6. Wang XY, Chen Y, Tang XJ, Jiang LH and Ji P: AMD3100 attenuates matrix metalloprotease-3 and -9 expressions and prevents cartilage degradation in a monosodium iodo-acetate-induced rat model of temporomandibular osteoarthritis. J Oral Maxillofac Surg 74: 927.e1-927.e13, 2016.

7. Walsh DA, Bonnet CS, Turner EL, Wilson D, Situ M and McWilliams DF: Angiogenesis in the synovium and at the osteochondral junction in osteoarthritis. Osteoarthritis Cartilage 15: 743-751, 2007.
8. Tibesku CO, Daniilidis K, Skwara A, Paletta J, Szuwart T and Fuchs-Winkelmann S: Expression of vascular endothelial growth factor on chondrocytes increases with osteoarthritis - an animal experimental investigation. Open Orthop J 5: 177-180, 2011.

9. Sun Y, Jin K, Childs JT, Xie L, Mao XO and Greenberg DA Vascular endothelial growth factor-B (VEGFB) stimulates neurogenesis: evidence from knockout mice and growth factor administration. Dev Biol 289: 329-335, 2006.

10. Walsh DA, McWilliams DF, Turley MJ, Dixon MR, Fransès RE, Mapp PI and Wilson D: Angiogenesis and nerve growth factor at the osteochondral junction in rheumatoid arthritis and osteoarthritis. Rheumatology (Oxford) 49: 1852-1861, 2010.

11. Jansen H, Meffert RH, Birkenfeld F, Petersen W and Pufe T: Detection of vascular endothelial growth factor (VEGF) in moderate osteoarthritis in a rabbit model. Ann Anat 194: 452-456, 2012.

12. Fazaeli S, Ghazanfari S, Everts V, Smit TH and Koolstra JH: The contribution of collagen fibers to the mechanical compressive properties of the temporomandibular joint disc. Osteoarthritis Cartilage 24: 1292-1301, 2016.

13. Morjen M, Honoré S, Bazaa A, Abdelkafi-Koubaa Z, Ellafi A, Mabrouk K, Kovacic H, El Ayeb M, Marrakchi N and Luis J: PIVL, a snake venom Kunitz-type serine protease inhibitor, inhibits in vitro and in vivo angiogenesis. Microvasc Res 95: 149-156, 2014.

14. Zhang S, Cao W, Wei K, Liu X, Xu Y, Yang C, Undt G, Haddad MS and Chen W: Expression of VEGF-receptors in TMJ synovium of rabbits with experimentally induced internal derangement. Br J Oral Maxillofac Surg 51: 69-73, 2013.

15. Wang YL, Li XJ, Qin RF, Lei DL, Liu YP, Wu GY, Zhang YJ, Yan-Jin, Wang DZ and Hu KJ: Matrix metalloproteinase and its inhibitor in temporomandibular joint osteoarthrosis after indirect trauma in young goats. Br J Oral Maxillofac Surg 46: 192-197, 2008.

16. Fransès RE, McWilliams DF, Mapp PI and Walsh DA: Osteochondral angiogenesis and increased protease inhibitor expression in OA. Osteoarthritis Cartilage 18: 563-571, 2010. 